19.Puchtler, H., F.S. Waldrop, S.N. Meloan, M.S. Terry, and H.M. Conner. 1970. Methacarn (methanol-Carnoy) fixation. Practical and theoretical considerations. Histochemie 21:97-116.

20.Kovarova, H., M. Hajduch, G. Korinkova, P. Halada, S. Krupickova, A. Gouldsworthy, N. Zhelev, and M. Strnad. 2000. Proteomics approach in classifying the basic of the anticancer activity of the new olomoucine-derived synthetic cyclin-dependent kinase inhibitor, bohemine. Electrophoresis 21:3757-3764.

21.Hajduch, M., Z. Kolar, R. Novotny, J. Hanus, V. Mihal, A. Hlobilkova, V. Noskova, and M. Strnad. 1997. Induction of apoptosis and regression of spontaneous dog melanoma following in vivo application of synthetic cyclin-dependent kinase inhibitor olomoucine. Anticancer Drugs 8:1007-1013.

22.Hajduch, M., L. Havlicek, J. Vesely, R. Novotny, V. Mihal, and M. Strnad. 1999. Synthetic cyclin dependent kinase inhibitors: new generation of potent anti-cancer drugs. Adv. Exp. Med. Biol. 457:341-353.

23.Verhaegen, S., A.J. McGowan, A.R. Brophy, R.S. Fernandes, and T.G. Cotter. 1995. Inhibition of apoptosis by antioxidants in the human HL-60 leukemia cell line. Biochem. Pharmacol. 50:1021-1029.

24.Junqueira, L.C., J. Carneiro, and R.O. Kelley. 1992. Basic Histology, 7th ed. PrenticeHall International, East Norwalk, CT.

25.McCloskey, T.W., S. Chavan, S.M. Lakshmi Tamma, and S. Pahwa. 1998. Comparison of seven quantitative assays to assess lymphocyte cell death during HIV infection: measurement of induced apoptosis in anti-Fas-treated Jurkat cells and spontaneous apoptosis in peripheral blood mononuclear cells from children infected with HIV. AIDS Res. Hum. Retroviruses 14:413-1422.

Received 19 February 2003; accepted 21 May 2003.

Address correspondence to Jirina Prochazkova, Department of Histology and Embryology, Medical Faculty of Palacky University, 77515 Olomouc, Czech Republic.e-mail:jipro@tunw.upol.cz.

\title{
Purification of Mycobacterium leprae RNA for gene expression analysis from leprosy biopsy specimens
}

\author{
Diana L. Williams, Sandra Oby-Robinson, Tana L. Pittman, \\ and David M. Scollard \\ National Hansen's Disease Programs at SVM, LSU, Baton Rouge, LA, USA
}

BioTechniques 35:534-541 (September 2003)

Gene expression analysis in Mycobacterium leprae, an obligate intracellular pathogen and the etiologic agent of leprosy, has been hampered by the lack of an efficient method to purify RNA from leprosy lesions. Therefore to date, transcripts for only a few genes have been identified. We report the use of a single-tube homogenization/RNA extraction method that produces enough RNA to study the expression of 30 genes from a single skin biopsy specimen of a multibacillary leprosy patient and demonstrate that RNA can be purified after fixation of biopsies in $70 \%$ ethanol for up to a year. This represents a major advancement in the ability to study M. leprae gene expression directly from biopsy material and should help to define genes that are associated with intracellular survival of this human pathogen.

\section{INTRODUCTION}

Mycobacterium leprae, the etiologic agent of leprosy, affects the skin, eyes, and mucosa of the upper respiratory tract and is unique among bacterial pathogens in its ability to invade the peripheral nervous system (1). Approximately 3 million leprosy patients in the world are physically disabled as a result of damage to peripheral nerves and the attendant sensorimotor loss. One-fourth of the 700,000 newly diagnosed patients each year (2) will suffer from irreversible nerve damage and are at risk of developing classic hand and foot deformities and associated disabilities as a long-term consequence of infection with M. leprae (3).

Fundamental to understanding the pathogenicity of $M$. leprae is to define the composite phenotype of the bacillus and to determine gene expression related to intracellular survival and virulence. A major obstacle impeding progress in this area has been our inability to culture $M$. leprae in vitro. Lacking sufficient quantities of viable $M$. leprae from biopsy material of leprosy lesions from which to study the biochemical basis of phenotype and genotype, most findings in this area have been of limited scope. In addition, gene expression analysis of $M$. leprae has been greatly hampered by the lack of a suitable method to purify RNA from infected tissues in sufficient quantities to analyze a large number of genes from a single biopsy. To date, transcripts for only a few $M$. leprae genes have been identified $(4,5)$. Therefore, there is a general lack of understanding of which genes are required for intracellular survival and virulence.

A major reason for the inability to purify $M$. leprae RNA from clinical specimens is that $M$. leprae possesses a complex mycobacterial surface structure consisting of a Gram-positive-like cell wall surrounded by a waxy, lipid, mycolic acid- and phenolic glycolipidcontaining envelope (6). This dense coat makes lysis of $M$. leprae for subsequent purification of RNA extremely difficult. Therefore, conventional techniques for purification of mycobacterial RNA for gene expression analysis have not been successfully applied to generate sufficient quantities of $M$. leprae mRNA, even when large numbers of bacteria from infected mouse foot pads have been extracted $(4,5)$. To complicate this issue, only low numbers of bacilli $\left(<10^{9}\right)$ are recovered from skin biopsies of leprosy lesions (7). Recently, we have described a method to purify RNA from purified bacteria, which employs a single-tube homogenization/RNA 
Table 1. Primers for RT-PCR Analysis of Mycobacterium leprae RNA Purification Methods

\begin{tabular}{|c|c|c|}
\hline Gene Name & & Primer Sequence \\
\hline \multirow{2}{*}{ hsp18 } & MLhsp18-F & 5'-ATTCGTCGTCGAGTTCGACCTTCCT-3' \\
\hline & MLhsp18-R & 5'-CTTAGCTTGTTGCGCAAACAACAGT-3' \\
\hline \multirow{2}{*}{ gap } & MLgap-F & 5'-GGCTCTTGCGGTTCG-3' \\
\hline & MLgap-R & 5'-GCAGGTCAGAATGCG-3' \\
\hline \multirow{2}{*}{$\operatorname{sod} A$} & MLsodA-F & 5'-TGAGATCAACGAGATCCACC-3' \\
\hline & MLsodA-R & 5'-GCCCAGTTTACGACATTCC-3' \\
\hline \multirow{2}{*}{ gyrA } & MLgyrA-F & 5'-GCATCTCCAATGTAGAAGACC-3' \\
\hline & MLgyrA-R & 5'-CAGTTATCGTGACAACGACC-3' \\
\hline \multirow[t]{2}{*}{$r p o B$} & MLrpoB-F & 5'-CGTCGAGGCGATCACGCCGCAGACGC-3' \\
\hline & MLrpoB-R & 5'-GACCTCTAGCCCGGCACGCTCACG-3' \\
\hline \multirow{2}{*}{ folP } & MLfolP-F & 5'-GTCCAGCACGGCCTGGC-3' \\
\hline & MLfolP-R & 5'-CTGCTCGCCCACCAG-3' \\
\hline
\end{tabular}

extraction method using a vertical angular motion homogenizer and standard chloroform extraction and RNA precipitation (8). Using this technique, microgram quantities of RNA, sufficient to perform $>150$ reverse transcription PCR (RT-PCR) assays and cross-species DNA array analysis, were obtained from $1 \times 10^{10}$ purified nude mouse foot pad-derived $M$. leprae. This RNA provided the first comprehensive insight into transcriptionally active genes that are potentially essential for intracellular survival of $M$. leprae. How- ever, when this procedure was applied to purify RNA from $M$. leprae obtained by standard Mickle homogenization of skin biopsy tissue of leprosy patients, only small amounts of $M$. leprae RNA were recovered (8). Therefore, to increase these RNA yields, we modified the above method by simply mincing biopsy material prior to the single-tube homogenization/RNA extraction method (direct method). In addition, since ethanol has been shown to preserve $M$. leprae DNA (9) and prokaryote RNA (10) for subsequent extraction method-

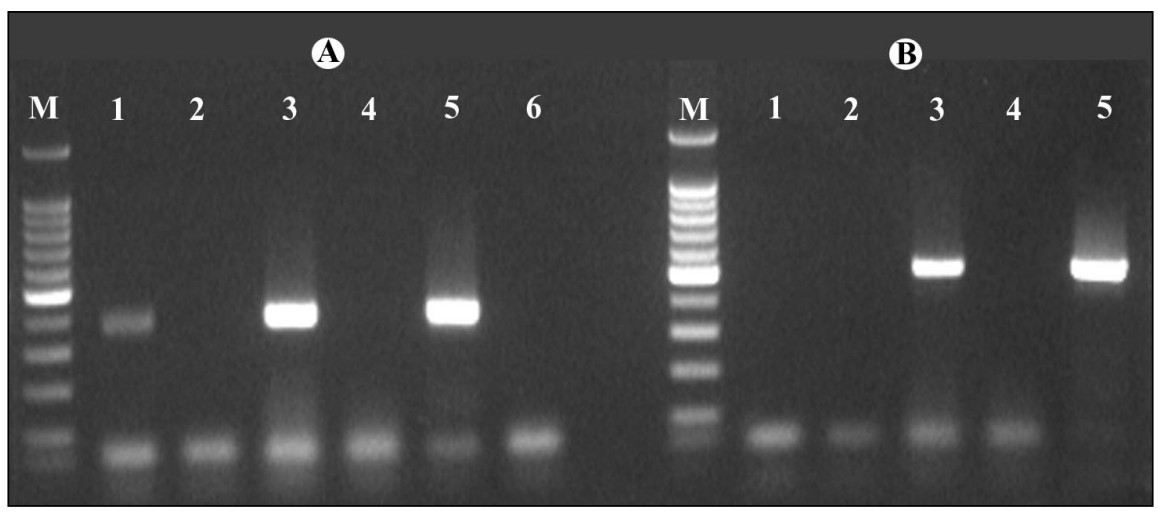

Figure 1. RT-PCR analysis of Mickle and direct method for purifying Mycobacterium leprae RNA from biopsy specimens of lepromatous leprosy patients. (A) $M$. leprae hsp 18 RT-PCR, generating a 360-bp fragment of the $h s p 18$ gene transcript and (B) M. leprae gap RT-PCR, generating a 706-bp fragment of the gap gene transcript. (A and B) Lane M, 100-bp DNA ladder (Promega, Madison, WI, USA); lane 1, M. leprae cDNA from Mickle homogenization method; lane 2, RT-control of Mickle homogenization method; lane 3, M. leprae cDNA from the direct mincing method; lane 4, RT-control of the direct mincing method; lane 5, $1 \mathrm{ng}$ M. leprae T-53 control DNA (positive control); (A) lane 6, human monocyte cDNA (negative control).

ologies, we determined the effect of ethanol fixation on the above procedure.

\section{MATERIALS AND METHODS}

\section{Skin Biopsy Specimens}

Diagnostic skin biopsies, obtained from multibacillary (MB) and paucibacillary (PB) leprosy patients prior to treatment, were bisected, and one-half was immersed in cryoprotective medium (O.C.T.; Tissue Tek, Columbus, $\mathrm{OH}, \mathrm{USA}$ ), snap-frozen in liquid nitrogen, and stored at $-70^{\circ} \mathrm{C}$. The other portion was fixed in $10 \%$ neutral-buffered formalin overnight and embedded in paraffin. Sections $(4 \mu \mathrm{m})$ were stained with hematoxylin and eosin for histopathologic classification and with Fite's acid-fast stain for determination of the bacterial index (BI). Biopsy specimens were also obtained from MB lesions and stored in $1 \mathrm{~mL}$ of $70 \%$ ethanol for up to 1 year at room temperature. To conceal patient identification, as specified by approved protocols for research with archived material, identifying information for each specimen was blinded by assigning a random code number to each specimen.

\section{RNA Extraction and Purification}

Biopsies were thawed, removed from the O.C.T. medium, rinsed three times with $1 \mathrm{~mL}$ sterile phosphate-buffered saline (PBS; Sigma, St. Louis, MO, USA), and weighed. Ethanol-fixed biopsies were removed from ethanol, rehydrated for $20 \mathrm{~min}$ in $1 \mathrm{~mL}$ diethylpyrocarbonate (DEPC) water (Sigma), and then the water was decanted. Biopsies (10-12 $\mathrm{mg}$ ) were then finely minced to a uniform consistency with sterile curved scissors in $50 \mu \mathrm{L} \mathrm{PBS}$.

M. leprae RNA was extracted directly from minced biopsy tissue using a modification of a previously described singletube homogenization/RNA extraction protocol (8) or bacteria-purified by the Mickle homogenization method (7). Briefly, $1 \mathrm{~mL}$ TRIzOL ${ }^{\circledR}$ Reagent (Invitrogen, Carlsbad, CA, USA) was added to each minced biopsy or purified bacteria. Each suspension was then transferred to a FastRNATM Blue tube (FastRNA KitBlue; Qbiogene, Carlsbad, CA, USA). 
Table 2. RT-PCR Analysis of the Direct and Mickle Methods for Purification of Mycobacterium leprae RNA from Skin Biopsy Specimens from Multibacillary Leprosy Patients

\begin{tabular}{|lcc|}
\hline & \multicolumn{2}{c|}{ RT-PCR } \\
\cline { 2 - 3 } Gene & Direct Method & Mickle Method \\
\hline hsp18 & $2 / 2^{\mathrm{a}}$ & $1 / 2$ \\
gap & $2 / 2$ & $0 / 2$ \\
sodA & $2 / 2$ & $1 / 2$ \\
gyrA & $2 / 2$ & $0 / 2$ \\
rpoB & $2 / 2$ & $0 / 2$ \\
folP & $2 / 2$ & $0 / 2$ \\
aNumber of biopsies positive of two biopsies \\
prepared by each method.
\end{tabular}

The tubes were processed in a vertical angular motion homogenizer (FastPrep ${ }^{\circledR}$ FP120 Instrument; Qbiogene) for $45 \mathrm{~s}$ at a speed setting of 6.5 and then allowed to sit for $5 \mathrm{~min}$ to cool. This procedure was repeated once. Tubes were held on ice for $5 \mathrm{~min}$, and $200 \mu \mathrm{L}$ chloroform/isoamyl alcohol (CIA) (24:1) (Sigma) were added. The tubes were then vortex mixed for 1 min. The FastRNA Blue glass matrix was pelleted at $700 \times g$ for $5 \mathrm{~min}$ at room temperature, and the entire liquid phase from each tube was transferred to a new 1.5-mL tube. Tubes were centrifuged at $14,000 \times g$ for $10 \mathrm{~min}$, and the aqueous phase from each tube was transferred to a new $1.5-\mathrm{mL}$ tube. A $100-\mu \mathrm{L}$ aliquot of Cleanascite ${ }^{\mathrm{TM}} \mathrm{HC}$ (CPG Biotech, Lincoln Park, NJ, USA) was added to each tube, and the tubes were mixed on a rocker shaker for $10 \mathrm{~min}$ to remove excess lipid material. Cleanascite was pelleted at $14,000 \times g$ for $1 \mathrm{~min}$ at room temperature, and each supernatant was added to a $1.5-\mathrm{mL}$ tube containing 500 $\mu \mathrm{L}$ CIA and vortex mixed for $10 \mathrm{~s}$. The phases were separated by centrifugation at $14,000 \times g$ for $2 \mathrm{~min}$, and the aqueous phase was transferred to a new $1.5-\mathrm{mL}$ tube, leaving $15 \%$ above the interface undisturbed. GlycoBlue ${ }^{\mathrm{TM}}$ co-precipitant (Ambion, Austin, TX, USA) $(1 \mu \mathrm{L})$ and one-tenth volume of $5 \mathrm{M} \mathrm{NH}_{4} \mathrm{OAc}$ were added, and the tubes were mixed. An equal volume of cold isopropanol was then added, and the tubes were mixed and then stored at $-80^{\circ} \mathrm{C}$ for at least 16 $\mathrm{h}$ to precipitate RNA. Tubes were then spun at $14,000 \times g$ for $30 \mathrm{~min}$ at $4^{\circ} \mathrm{C}$, and the RNA pellets were washed in $500 \mu \mathrm{L}$ of salt ethanol wash solution (SEWS) (from the FastRNA Kit-Blue), air-dried at room temperature for $5 \mathrm{~min}$, and dissolved in $35 \mu \mathrm{L}$ of DEPCtreated water. DNA was removed from RNA preparations using the DNA-free $e^{\mathrm{TM}}$ kit (Ambion), as specified by the manufacturer, and purified RNA was stored at $-80^{\circ} \mathrm{C}$.

To provide a positive control for all RT-PCR analyses, DNA was purified from 1 $\times 10^{10}$ M. leprae Thai-53 freshly harvested from infected nude mouse foot pad tissue using a previously described procedure (8).

\section{Reverse Transcription}

cDNA was made from $10 \mu \mathrm{L}$ of $M$. leprae RNA using the Advantage ${ }^{\mathrm{TM}}$ cDNA Polymerase Mix, Advantage RTfor-PCR Kit, and random hexamers in a final volume of $50 \mu \mathrm{L}$ according to the manufacturer's recommendations (BD Biosciences Clontech, Palo Alto, CA, USA). Controls for DNA contamination consisted of: $M$. leprae RNA, incubated with the reverse transcription reaction reagents excluding the Moloney murine leukemia virus (MMLV)-RT (RT control); and cDNA from human monocyte RNA and $M$. leprae T-53 DNA (PCR specificity controls).

\section{PCR and DNA Sequencing}

PCR assays were initially developed using gene-specific primers (Table 1) and $M$. leprae T-53 DNA for six genes, representing several different $M$. leprae gene families (http://www. sanger.ac.uk/ Projects/M_leprae/). These genes included: (i) hspl8, encoding the $18 \mathrm{kDa}$ heat shock protein, HSP 16.7 (chaperones/ heat shock); (ii) gap, encoding the glyceraldehyde 3-phosphate dehydrogenase (glycolysis); (iii) sodA, encoding the superoxide dismutase (detoxification); (iv) gyrA, encoding a putative DNA gyrase subunit A (DNA replication, repair, recombination, and restriction/ modification); (v) rpoB, encoding the $\beta$-subunit of RNA polymerase (RNA synthesis, RNA modification, and DNA transcription); and ( $v i$ ) folP, encoding the dihydropteroate synthase (folic acid biosynthesis). Then, $5 \mu \mathrm{L}$ of each $M$. leprae cDNA preparation and RT controls were amplified in 50- $\mu \mathrm{L}$ PCRs. The resultant PCR products were separated by agarose gel electrophoresis on $2 \% \mathrm{NuSieve}^{\circledR}$ GTG/SeaKem ${ }^{\circledR}$ GTG (1:1) agarose gels (BioWhitaker, Rockland, ME, USA) in $1 \times$ TAE buffer (4 M Tris-acetate, $\mathrm{pH}$ 8.0, 1 mM EDTA; Invitrogen). Ethidium bromide-stained gels were scanned using the GelDoc ${ }^{\circledR} 2000$ Instrument (Bio-Rad Laboratories, Hercules, CA, USA). The reproducibility and sensitivity of the direct homogenization/RNA extraction protocol were determined using $M$. leprae gap and hsp 18 PCR analysis with cDNA from $6 \mathrm{MB}$ and $6 \mathrm{~PB}$ leprosy patients. PCR products were purified using QIAquick $^{\mathrm{TM}}$ PCR Purification Kit spin columns (Qiagen, Valencia, CA, USA) according to manufacturer's recommendations. The DNA sequence of each PCR fragment was obtained using $2 \mu \mathrm{M}$ of forward primer for each gene fragment, PCR product, and automated dideoxy DNA sequencing (GeneLab, SVM, LSU, Baton Rouge, LA, USA).

\section{RESULTS AND DISCUSSION}

Initial results of this study demonstrated that $M$. leprae hspl8 and gap gene transcripts were detected in cDNA obtained from the direct single-tube homogenization/RNA extraction of a minced skin biopsy of a lepromatous leprosy patient but not from the RT control (Figure 1). In addition, lower levels of hsp18 transcripts and no gap transcripts were detected using the Mickle method. When the expression of these and four other genes was further analyzed from two MB skin biopsies processed by each method, gene transcripts were detected for all of the genes in the cDNA obtained from the direct method (Table 2). However, transcripts were only detected for hsp18 and sodA in the cDNA obtained from the Mickle method. These data suggested that there was a substantial loss of $M$. leprae RNA using the Mickle method. A plausible explanation for this is that large numbers of bacteria remain associated with the cellular debris, and 


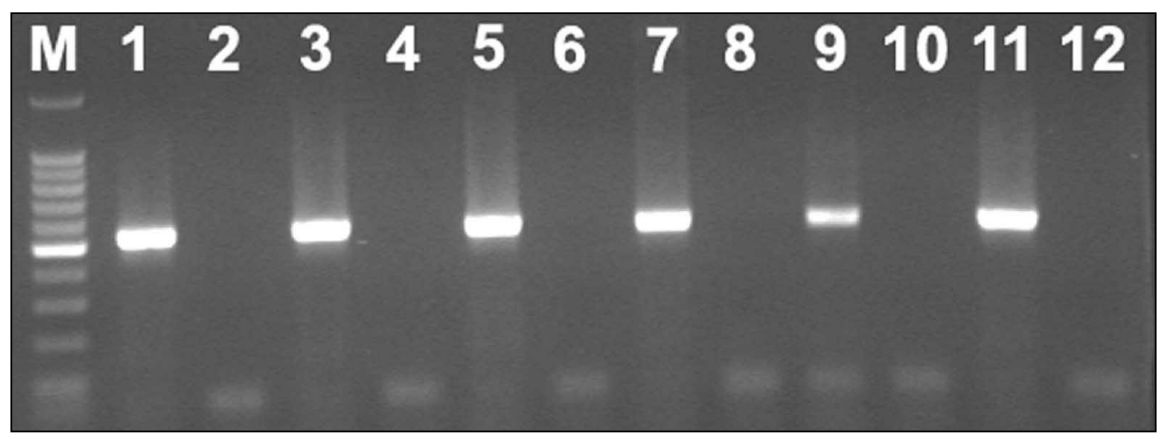

Figure 2. Mycobacterium leprae gap reverse transcription PCR (RT-PCR) analysis of $M$. leprae RNA purification from ethanol-fixed skin biopsies. Lane M, 100-bp DNA ladder (Promega); lane 1, cDNA from unfixed biopsy; lane 2, unfixed biopsy RT-control; lane 3, cDNA from $24 \mathrm{~h}$ ethanol-fixed biopsy; lane 4, $24 \mathrm{~h}$ ethanol-fixed biopsy RT-control; lane 5, cDNA from $48 \mathrm{~h}$ ethanol-fixed biopsy; lane 6, $48 \mathrm{~h}$ ethanol-fixed biopsy RT-control; lane 7, cDNA from 1 week ethanol-fixed biopsy; lane 8, 1 week ethanol-fixed biopsy RT-control; lane 9, cDNA from 1 year ethanol-fixed biopsy; lane 10, 1 year ethanol-fixed biopsy RT-control; lane11, $1 \mathrm{ng}$ M. leprae T-53 control DNA (positive control); lane 12, human monocyte cDNA (negative control).

since this debris is removed prior to RNA purification, many $M$. leprae are lost. We therefore concluded that direct homogenization/RNA extraction of finely minced biopsy tissues using an angular motion homogenizer and TRIzOL provides an improved method for simultaneously disrupting tissue, lysing both human and bacterial cells and extracting M. leprae RNA.

Using the direct method, $M$. leprae RNA was obtained from all MB skin biopsies containing BIs $>2+$ (Table 3 ), demonstrating the reproducibility of this method. Although this technique represents an improvement in M. leprae RNA yields from biopsies of MB leprosy patients compared to our previous procedure (8), no M. leprae RNA was detected in PB biopsies, $(\mathrm{BI} \leq 2+)$, and therefore, gene expression analysis of $M$. leprae cannot be performed on PB biopsies, representing the tuberculoid end of the leprosy spectrum, using this technique.

While our earlier report demonstrated the feasibility of obtaining $M$. leprae RNA from biopsy tissue of leprosy patients and has used this RNA to determine the expression of a few genes (8), the direct method produced $150 \mu \mathrm{L}$ cDNA, which is sufficient template to perform 30 individual 50- $\mu$ L RT-PCRs. It is therefore anticipated that combining the RNA from several MB biopsies will be sufficient to produce enough cDNA to study $>100 \mathrm{M}$. leprae genes using RT-PCR and potentially provide enough 
Table 3. Purification of Mycobacterium leprae RNA from Skin Biopsies of Leprosy Lesions Using the Direct Single-Tube Homogenization/RNA Extraction Method

\begin{tabular}{|lccc|}
\hline Biopsy No. & Bacterial Index & \multicolumn{3}{c|}{ RT-PCR } \\
\hline Multibacillary & & hsp18 & gap \\
\hline 10817 & $3-6+$ & + & + \\
16163 & $5-6+$ & + & + \\
72368 & $5+$ & + & + \\
71398 & $5+$ & + & + \\
75338 & $4-6+$ & + & + \\
49356 & $3-4+$ & + & + \\
Paucibacillary & & & - \\
91547 & $2+$ & - & - \\
56187 & $2+$ & - & - \\
38572 & $2+$ & - & - \\
46111 & $2+$ & - & - \\
82599 & $2+$ & - & - \\
99837 & $2+$ & & - \\
\hline
\end{tabular}

RNA for full genomic microarray analysis when this tool becomes available. Therefore, it is now possible to analyze the expression of a large number of $M$. leprae genes directly from biopsies obtained from MB leprosy patients. This represents a major advancement in the ability to study $M$. leprae gene expression directly from biopsy material and should help to define genes that are associated with intracellular survival of this human pathogen.

The present study also demonstrated that storage of skin biopsies in $70 \%$ ethanol efficiently preserved $M$. leprae RNA for up to 1 year for subsequent gene expression analysis (Figure 2). This is a significant finding, since there are very few tissue banks that contain well-characterized biopsies from leprosy patients, and therefore, ethanol-fixation of biopsies in the field and storage of these specimens at room temperature will provide a valuable source of specimens for $M$. leprae gene expression studies. In addition, since RNA transcription is terminated during ethanolfixation of tissue, fixation of biopsies immediately after specimen acquisition should give an accurate assessment of the specific gene transcripts required for intracellular survival of $M$. leprae.

In summary, this study describes the development and characterization of an improved method for the preservation and purification of $M$. leprae RNA directly from frozen or ethanol-fixed skin biopsies of leprosy lesions. It is anticipated that this method will provide highly purified $M$. leprae RNA to study gene expression from granulomatous lesions of leprosy patients and, thereby, help to identify the genes associated with intracellular survival and virulence of $M$. leprae. In addition, the availability of mRNA from $M$. leprae should assist to identify a suitable mRNA target for the development of a quantitative RT-PCR assay for M. leprae viability analysis.

\section{ACKNOWLEDGMENTS}

We would like to acknowledge Drs. Thomas Gillis, Kathleen Eisenach, and Issar Smith for their insightful discussions about procedural aspects of this manuscript. This study was supported by the Heiser Foundation for Leprosy and Tuberculosis Research and The
Division of the National Hansen's Disease Programs, the Department of Health and Human Services (DHHS), the Health Resource and Service Administration (HRSA), and the Bureau of Primary Health Care (BPHC).

\section{REFERENCES}

1.Pfaltzgraff, R.E. and A. Bryceson. 1985. Clinical Leprosy, p. 134-176. In R.C. Hastings (Ed.), Leprosy, 1st ed. Churchill Livingstone, New York.

2.World Health Organization. Leprosy situation by WHO regions end of 2000. WHO Weekly Epidemiological Record No. 77. (http://www.who.int/lep/12.html).

3.Clements, B.R. and D.M. Scollard. 1996 Leprosy, p. 9.1-9.28. In G.L. Mandell and R. Fekety (Eds.), Atlas of Infectious Diseases, Vol VIII. Current Medicine, Philadelphia.

4.Patel, B.K, D.K. Banerjee, and P.D. Butcher. 1993. Determination of Mycobacterium leprae viability by polymerase chain reaction amplification of 71-kDa heat-shock protein mRNA. J. Infect. Dis. 168:799-800.

5.Williams, D.L., L. Spring, E. Harris, P. Roche, and T.P. Gillis. 2000. The dihydropteroate synthase of Mycobacterium leprae and dapsone resistance. Antimicrob. Agents Chemother. 44:1530-1537.

6.Marques, M.A., S. Chitale, P.J. Brennan, and M.C. Pessolani. 1998. Mapping and identification of the major cell-wall associated components of Mycobacterium leprae. Infect. Immun. 66:2625-2631.

7.Rees, R.J. 1984. The microbiology of leprosy, p. 31-52. In R.C. Hastings (Ed.), Leprosy, 1st ed. Churchill Livingstone, New York.

8.Williams, D.L., S. Oby-Robinson, T.L. Pittman, M.T. Torrero, R. Truman, M. Yoder, D. Geiman, N. Morrison, et al. Gene Expression in Mycobacterium leprae: defining a partial gene set for survival of this unique pathogen. Int. J. Lepr. (In press).

9.Fiallo, P., D.L. Williams, G. Chan, and T.P. Gillis. 1992. Effects of fixation on polymerase chain reaction detection of Mycobacterium leprae. J. Clin. Microbiol. 30:3095-3098.

10.Bachoon, D.S., F. Chen, and R.E. Hodson. 2001. Recovery and detection of mRNA by RT-PCR from preserved prokaryotic samples. FEMS Microbiol. Lett. 201:127-132.

Received 21 February 2003; accepted 4 June 2003.

Address correspondence to Diana L. Williams, Molecular Biology Research Department, Laboratory Research Branch, National Hansen's Disease Programs at LSU-SVM, Rm. 3517W, Skip Bertman Drive, Baton Rouge, LA 70803, USA. e-mail:dwill21@lsu.edu 